\title{
Quantitative evaluation of atherosclerotic plaques using cross- polarization optical coherence tomography, nonlinear, and atomic force microscopy
}

Ekaterina V. Gubarkova

Mikhail Yu. Kirillin

Varvara V. Dudenkova

Peter S. Timashev

Svetlana L. Kotova

Elena B. Kiseleva

Lidia B. Timofeeva

Galina V. Belkova

Anna B. Solovieva

Alexander A. Moiseev

Gregory V. Gelikonov

Ilya I. Fiks

Felix I. Feldchtein

Natalia D. Gladkova 


\title{
Quantitative evaluation of atherosclerotic plaques using cross-polarization optical coherence tomography, nonlinear, and atomic force microscopy
}

\author{
Ekaterina V. Gubarkova, ${ }^{a, *}$ Mikhail Yu. Kirillin, ${ }^{b}$ Varvara V. Dudenkova, ${ }^{a, c}$ Peter S. Timashev, ${ }^{\text {dee }}$ \\ Svetlana L. Kotova, ${ }^{f}$ Elena B. Kiseleva, ${ }^{a}$ Lidia B. Timofeeva, ${ }^{a}$ Galina V. Belkova, ${ }^{f}$ Anna B. Solovieva, ${ }^{f}$ \\ Alexander A. Moiseev, ${ }^{\mathrm{b}}$ Gregory V. Gelikonov, ${ }^{\mathrm{b}}$ Ilya I. Fiks, ${ }^{\mathrm{b}}$ Felix I. Feldchtein, ${ }^{\mathrm{a}}$ and Natalia D. Gladkova ${ }^{\mathrm{a}}$ \\ aNizhny Novgorod State Medical Academy, 10/1 Minin and Pozharsky Square, Nizhny Novgorod 603950, Russia \\ Institute of Applied Physics RAS, 46 Ulyanov Street, Nizhny Novgorod 603950, Russia \\ 'N.I. Lobachevsky State University of Nizhny Novgorod, 23 Gagarin Avenue, Nizhny Novgorod 603950, Russia \\ Institute of Photonic Technologies, Research Center of Crystallography and Photonics RAS, 2 Pionerskaya Street, Troitsk, Moscow 142190, \\ Russia \\ I.M. Sechenov First Moscow State Medical University, 2-4 Bolshaya Pirogovskaya Street, Moscow 119991, Russia \\ ${ }^{f}$ N.N. Semenov Institute of Chemical Physics, 4 Kosygin Street, Moscow 119991, Russia
}

\begin{abstract}
A combination of approaches to the image analysis in cross-polarization optical coherence tomography (CP OCT) and high-resolution imaging by nonlinear microscopy and atomic force microscopy (AFM) at the different stages of atherosclerotic plaque development is studied. This combination allowed us to qualitatively and quantitatively assess the disorganization of collagen in the atherosclerotic arterial tissue (reduction and increase of CP backscatter), at the fiber (change of the geometric distribution of fibers in the second-harmonic generation microscopy images) and fibrillar (violation of packing and different nature of a basket-weave network of fibrils in the AFM images) organization levels. The calculated CP channel-related parameters are shown to have a statistically significant difference between stable and unstable (also called vulnerable) plaques, and hence, CP OCT could be a potentially powerful, minimally invasive method for vulnerable plaques detection. () 2016 Society of Photo-Optical Instrumentation Engineers (SPIE) [DOI: 10.1117/1.JBO.21.12.126010]
\end{abstract}

Keywords: cross-polarization optical coherence tomography; cross-polarization backscatter; second-harmonic generation; atomic force microscopy; atherosclerotic plaque; collagen fibers.

Paper 160555PR received Aug. 16, 2016; accepted for publication Nov. 28, 2016; published online Dec. 20, 2016.

\section{Introduction}

The main cause of coronary heart disease is currently considered to be related to atherosclerosis of the coronary arteries with the deposition of cholesterol plaques (atherosclerotic plaques) in them and narrowing of the arterial lumen. The acute coronary syndrome is most often caused by the rupture of a vulnerable plaque, which is characterized histologically by the presence of necrotic, lipid-rich core at the bottom, and a thin $(<65 \mu \mathrm{m})$ fibrous cap. ${ }^{1}$ Moreover, the plaque instability affects proteolysis of coating fibrous collagen by metalloproteinases (released by activated macrophages) and smooth muscle cells apoptosis, thus inhibiting the collagen synthesis and further weakening the fibrous cap. ${ }^{2-4}$ When these plaques rupture, they release a thrombogenic necrotic material into the blood resulting in a vessel occlusion. ${ }^{5}$ Therefore, a need exists for minimally invasive detection of early signs of intimal thickening and plaque vulnerability, which can be facilitated by improved techniques for assessing the structure and composition of atherosclerotic plaques.

Polarization-sensitive (PS) optical coherence tomography (OCT) is a minimally invasive imaging modality enabling evaluation of the collagen concentration and can potentially enhance the possibility of in vivo plaque detection. ${ }^{6-8}$ As shown in Ref. 6, the birefringence of fibrous and fibro-calcified plaques, the

*Address all correspondence to: Ekaterina V. Gubarkova, E-mail: kgybarkova@mail.ru dominant extracellular component of which is organized as thick collagen bundles, was significantly higher than the birefringence for a normal vascular wall with only a small amount of thin collagen fibers. However, the approach relying on birefringence alone does not allow differentiation of the important plaque development stages. In particular, the hallmark feature of vulnerable plaques having a serious risk of rupture is the presence of a lipid core. In addition, the small cap thickness of a vulnerable plaque (less than $\sim 65 \mu \mathrm{m}$ ) is not sufficient to accumulate measurable phase retardation (phase delay between eigen polarizations).

This study used a cross-polarization (CP) OCT system, and the OCT images were created in two channels, one of which was copolarized with the incident polarization and the other one was orthogonal (or, in other words, cross-polarized) to the incident polarization, respectively. ${ }^{9}$ While CP OCT is considered as a particular case of PS OCT, the focus in CP OCT is typically placed on the information contained in the CP channel whereas in the "traditional" PS OCT the focus is on the birefringence/ phase retardation or other polarization parameters (such as the fast axis orientation) map. Typically, in PS OCT the CP channel is acquired but not even visualized. In this study, we evaluate both CP OCT images and effective birefringence information but still call it CP OCT, partially for historical reasons 
and connection with the previous publications using the same or similar systems.

From the light-tissue interaction prospective, there are at least two different mechanisms responsible for the CP OCT image. One is the tissue birefringence. It occurs if optical anisotropy exists, resulting in polarization changes because of a different light speed for two orthogonally polarized eigen light components.

Another mechanism is coherent $\mathrm{CP}$ backscattering. It was described in-depth by Schmitt and Xiang ${ }^{10}$ including a theoretical description of optical scattering on nonspherical particles and particles with sizes much larger than a wavelength.

In the presence of strong vertical gradients in the OCT images, it is not easy or even possible to separate the contributions of these two mechanisms. In some cases, the information about the tissue microstructure obtained from histology and other modalities can help with separating them in CP OCT. In particular, for cardiovascular applications, the main sources of strong cross-polarized backscattering in the intima/fibrous cap of a plaque include collagen fibers and large elongated foam cells of inflammation. ${ }^{11}$ This study represents an attempt to move from qualitative visualization, such as the one in Ref. 11, toward an objective extraction of the features using quantitative image processing. Two quantitative parameters extracted from CP OCT are the depolarization factor (DF) and the effective birefringence (detailed description is given below in Sec. 2.3). While the effective birefringence formula looks exactly like a commonly used birefringence calculation in traditional PS OCT, it can only be interpreted as a real birefringence in the case of homogeneous anisotropic media, free from strong coherence backscatter (such as cartilage, tendon, or muscle). In more complex tissues, such as vascular walls, normal, or containing plaques, these parameters, as will be shown below, still may be useful for plaque differentiation; however, they should be mostly considered as evidence-based parameters. The direct association of the DF and effective birefringence, on one side, and coherent backscattering and real birefringence, on the other side, is challenging and may be misleading.

Nonlinear microscopy, including second-harmonic generation (SHG) and two-photon-excitation fluorescence (TPEF) with spectral detection, has the ability to visualize the extracellular matrix of unstained coronary arterial walls ex vivo, revealing the distribution of collagen and elastin fibers. ${ }^{12-14}$ Recent publications report the development of algorithms for automatic quantitative assessment of collagen fibers in the SHG microscopy images of the rabbit aorta using fast Fourier transformation (FFT) and the gray level co-occurrence matrix calculations. ${ }^{13,14}$ However, this method application for differentiation and detailed analysis of the plaque development stages has not been established.

Atomic force microscopy (AFM) is used to study spatial collagen organization with the nanometer scale spatial resolution, which is superior to optical microscopy. The characteristic packing of collagen fibrils and fibers was obtained for the walls of coronary arteries affected by atherosclerosis, in particular, for those with vulnerable plaques. ${ }^{15}$ Furthermore, the analysis of the surface roughness along with the use of flicker-noise spectroscopy (FNS) parameterization allowed quantification of the nanoscale surface morphology visualized by AFM. ${ }^{15,16}$

In this study, high-resolution nonlinear microscopy and AFM were used for the comparison and correlation with the findings of CP OCT. Of these three modalities, only CP OCT currently can be translated to an affordable clinical use. The comparison with the other methods is intended to verify and validate CP OCT for the future in vivo use in the clinical setting. In particular, the purpose of this study is to develop an approach for quantitative evaluation of changes in the polarization properties in the intima/ fibrous cap, to characterize and differentiate the atherosclerotic plaques development stages, and to assess their instability.

\section{Materials and Methods}

\subsection{Human Arterial Samples}

This study was performed on 30 postmortem samples of coronary artery walls evaluated in parallel by CP OCT, histology, nonlinear microscopy, and AFM. The samples were obtained during autopsy in accordance with a scientific research protocol approved by the Research Ethics Board of the Nizhny Novgorod State Medical Academy, Russia. All arterial vessels were obtained no later than $24 \mathrm{~h}$ after death and delivered to the laboratory in gauze saturated with the phosphate buffer at $7^{\circ} \mathrm{C}$.

CP OCT imaging was performed from the intimal side of the samples. After the OCT imaging, the OCT probe area $(6 \mathrm{~mm}$ in the diameter) on the sample surface was marked with an ink circle and the sample was fixed (10\% formalin for $48 \mathrm{~h}$ with the standard paraffin embedding process) and serially sliced in the central area, with the slice orientation matching the OCT imaging plane. Serial $10-\mu$ m-thick sections were used for nonlinear microscopy, $7-\mu \mathrm{m}$-thick sections were used for AFM and histological slides stained with hematoxylin and eosin (H\&E). A total of 108 sets of CP OCT, histology, nonlinear microscopy, and AFM images were acquired and analyzed. Among these 108 sets, we found lesions of the following different degrees of atherosclerosis development: 19 thickened intima (stage I), 8 thickened intima with a lipid streak (stages II/III), 9 fibrous cap over a small lipid core (stage IV), 19 thin fibrotic cap over a big lipid core (stage Va, a most interesting one from the clinical viewpoint), 32 fibro-calcified plaque (stage $\mathrm{Vb}$ ), and 21 very thick fibroatheromas (stage $\mathrm{Vc}) .{ }^{17}$

\subsection{Cross-Polarization Optical Coherence Tomography System}

The spectral domain CP OCT system used in the study has been described in Refs. 11, 18, and 19. The light source was a superluminescent diode with a central wavelength of $1310 \mathrm{~nm}$, radiation power of $20 \mathrm{~mW}$, and a spectral width of $100 \mathrm{~nm}$, resulting in the axial resolution of $15 \mu \mathrm{m}$, scanning depth of $1.7 \mathrm{~mm}$, scanning speed of 20,000 A-scans per second. The B-scans were formed from 512 consecutive A-scans with the frame rate of 40 frames/s and transverse scanning range of $2.7 \mathrm{~mm}$ for both images in the co- and cross-polarization channels. The spectrometer uses a prism-based linearization of the spectrum in the $k$-space. ${ }^{18,20}$ Additional linearization was done with a signal processing as described in Ref. 19. As per common path interferometer configuration utilized here, there are no separate sample and reference arms. The reference radiation is created by a partial reflector in the tissue probe, and the primary Fizeau interferometer is created between this partial reflection and the actual tissue backscattering. The additional interferometer (upper part of Fig. 1) has its optical path difference approximately equal to the primary interferometer offset $L_{0}$. One arm of the additional interferometer contains an electrically controlled 45 deg Faraday rotator. For the copolarization channel, 


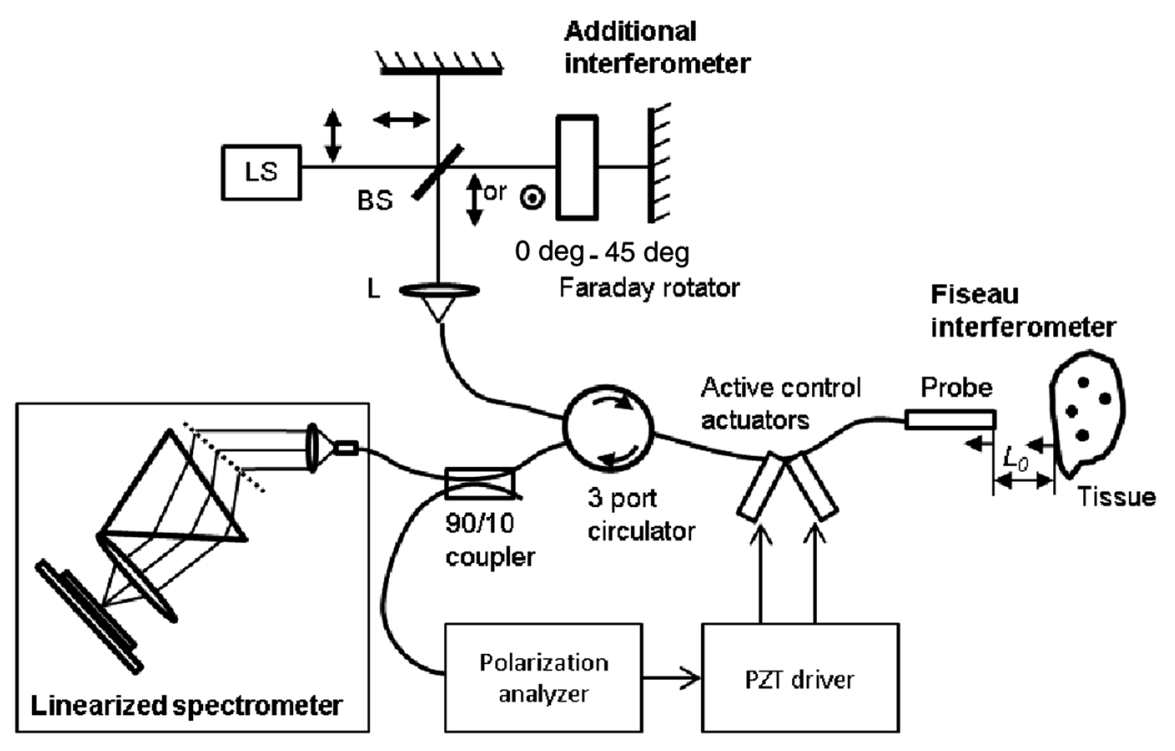

Fig. 1 CP OCT schematic. LS, light source; BS, beam splitter; L, lens; and $L_{0}$, interferometer offset. Reprinted, with permission, from Gubarkova et al. ${ }^{11}$

no driving voltage is applied, so polarization states in both arms are coaligned linear. Application of driving voltage rotates the polarization state by 90 deg and yields the orthogonal polarization states in the two arms, corresponding to the CP channel. The co- and cross-polarization channels are acquired sequentially in the left-to-right and right-to-left lateral scanning directions. The system had an active, closed loop control of the polarization at the fiber output, enabling maintenance of the circular polarization state emerging from the tissue probe; this is true even in the presence of fiber mechanical bends and resultant changes in the fiber birefringence.

Note that the tissue optical anisotropy is related to its fiber orientation and the tissue eigen polarizations are linear. To observe the tissue polarization response, it is interrogated with the equal mix of the eigen linear polarizations. The circular polarization is best suited for this purpose, because it automatically provides this equal mix and does not require any alignments between the incident polarization and tissue anisotropy axes orientation. The system acquires the copolarization channel in odd-numbered (left-to-right) B-scans; then the Faraday rotator is activated to acquire $\mathrm{CP}$ channel in the even-numbered (right-to-left) B-scans.

\subsection{Quantitative Processing of the Cross-Polarization Optical Coherence Tomography Images}

The quantitative processing of the CP OCT images involved calculation of the DF and effective birefringence $(\Delta n)$.

The calculation algorithm for DF was developed previously and applied for the evaluation of the collagen fibers' state in a variety of tissues (bladder mucosa and aorta). ${ }^{21,22}$ The DF is calculated as a ratio of OCT signals in the cross- and co-polarization channels according to the following equation:

$\operatorname{DF}(x, z)=\frac{I(x, z)^{\perp}-\left\langle I_{\text {noise }}\right\rangle}{I^{\|}(x, z)}$,

where $I^{\perp}(x, z)$ and $I^{\|}(x, z)$ are the raw linear (not logarithmic) OCT signals in the cross- and co-polarizations in a specific pixel $(x, z)$ after adjacent averaging over a $5 \times 5$ pixel area. The DF is calculated for those pixels where the OCT signal in the CP exceeds the average value of noise $\left(I_{\text {noise }}\right)+$ double noise standard deviation (SD) $2 \sigma$. The system noise is calculated from the OCT image in the areas where the useful signal is not noticeable.

Maps of effective birefringence $(\Delta n)$ were also built for CP OCT images as the in-depth derivative of the effective phase delay, as suggested in Ref. 6, similar to mapping the birefringence coefficient for PS OCT images and expressed according to the following equation:

$\Delta n(x, z)=\frac{1}{k_{0}} \frac{\partial}{\partial z} \operatorname{arctg}\left[\frac{I^{\perp}(x, z)}{I^{\|}(x, z)}\right]$,

where $I^{\perp}(x, z)$ and $I^{\|}(x, z)$ are the raw linear (not logarithmic) OCT signals in the cross- and co-polarization channels, respectively, and $k_{0}$ is a wavevector magnitude.

This approach considers the phase delay and the resulting birefringence as effective values, because both processes-birefringence and $\mathrm{CP}$ backscatter - take part in the formation of the CP OCT signal and are hard to separate.

These values were also averaged over selected regions of interest (ROI) as the integral DF (IDF) and the average birefringence, respectively. As discussed earlier in Sec. 1, while it may be tempting to associate the IDF with the CP backscatter and effective $\Delta n$ with the tissue birefringence, this should be attempted with great caution.

It should be noted that the terminology difference for the same spatial averaging (using the words "integral" and "average") happens for historical reasons because the IDF was already used in the previous publications ${ }^{21,22}$ while the term "average birefringence" has been used in the literature for a long time. ${ }^{23}$

\subsection{Average Isotropy Index for Nonlinear Microscopy Images}

Visualization of collagen and elastic fibers in the vessels' walls was conducted with an LSM 710 confocal laser-scanning microscope (Carl Zeiss, Germany). A pulsed Ti:sapphire laser 
(Chameleon Vision2, Coherent, Santa Clara) emitting 140 fs pulses with a central wavelength of $800 \mathrm{~nm}$ at a repetition rate of $80 \mathrm{MHz}$ was used. The signals were detected simultaneously in two channels using filters in the wavelength ranges of 362 to $415 \mathrm{~nm}$ and 480 to $554 \mathrm{~nm}$, when detecting the SHG signal from collagen fibers and the TPEF signal from elastin, respectively. ${ }^{24}$ The images were obtained using a C-Apochromat waterimmersion objective with a $40 \times$ magnification and a numerical aperture of 1.2 , providing a $212 \times 212 \mu \mathrm{m}$ field of view $(1024 \times 1024$ pixels $)$.

The quantitative processing of the nonlinear microscopy images was performed based on Fourier analysis. ${ }^{13,24}$ The two-dimensional Fourier spectrum of the images was binarized with the threshold level of $30 \%$ of the maximum pixel intensity. At the next step, the ratio of the smaller to the larger ellipse axes was calculated. This ratio is called the average isotropy index and could serve as a measure of the tissue's anisotropy. Structures with the fiber orientation along a certain prevailing direction tend to have a smaller value of the isotropy index. Disorganization of fibers with a more isotropic distribution results in the isotropy index increasing toward 1 .

\subsection{Flicker-Noise Spectroscopy Parameterization of Atomic Force Microscopy Images}

The tissue sections on glass slides were imaged on air in the semicontact mode, using a Solver P47 AFM (NT-MDT, Russia), a Solver scanner $(14 \times 14 \times 2 \mu \mathrm{m})$, and tapping etched silicon probes (Bruker, Billerica, Massachusetts) with a nominal spring constant of $42 \mathrm{~N} / \mathrm{m}$, nominal resonant frequency of $320 \mathrm{kHz}$, and a nominal tip radius of $8 \mathrm{~nm}$. The acquisition of $6 \times 6 \mu \mathrm{m}$ AFM images was performed at a scan rate of $1 \mathrm{~Hz}$, with a $512 \times 512$ pixels resolution. The ROI for AFM was selected in accordance with the histological findings, with the use of an optical microscope combined with the AFM instrument.

The FNS technique was applied to quantitatively parameterize the morphology of the arterial wall's extracellular matrix and its changes occurring with the progression of atherosclerosis. FNS is a general phenomenological approach for analyzing series of chaotic signals, via the search of correlations in a sequence of dynamic variables and ascribing an informational significance to such correlations. FNS, as a technique of statistical physics, introduces a number of nanometrology parameters, which may quantitatively characterize the surface texture. ${ }^{16}$ Application of FNS instead of standard metrological parameters (ISO 4287) is highly preferable in case of biological samples characterized by a considerable height heterogeneity. While the standard metrology parameters are nonspecific and may reflect the entire history of a sample preparation, which affects its surface (pretreatment, drying, and microtome sectioning), FNS parameterization generally extracts only correlations inherent for the sample's structure, essentially filtering out the systematic artifacts brought in by the sample preparation.

The details of the FNS algorithm are described in Ref. 16. Briefly, when applying the FNS parameterization to the three-dimensional surface images obtained by AFM, the roughness profiles $h(x, y)$ are considered as the dynamic variables, and the extracted surface parameters are related to the autocorrelation function for every profile. Different ranges of spatial frequencies are considered in the $h(x, y)$ profiles: low-frequency ("resonant") and high-frequency ranges, the latter represented by two basic types of irregularities against the low-frequency baseline-_jumps" (relatively moderate-height steps) and "spikes" (very narrow peaks of a relatively significant height).

The contribution of the low-frequency baseline (originating from the sample positioning and other manipulations) is excluded, so only high-frequency random steps and spikes remain for consideration. In general, several FNS parameters are introduced, but, as was shown earlier, ${ }^{15}$ the changes in the biological samples' morphology are best characterized by the two basic FNS parameters reflecting contributions of the above-mentioned types of high-frequency irregularities: a "stepwiseness factor" $\sigma(\mathrm{nm})$ for the jump-like irregularities of the studied AFM-profile and a "spikiness factor" $S\left(L_{0}^{-1}\right)\left(\mathrm{nm}^{2} \mu \mathrm{m}\right)$ for the spike-like irregularities of the studied profile, where $L_{0}$ is the length of correlation for the high-frequency irregularities at the nanoscale (see in Ref. 16 for the corresponding calculation procedure).

A custom-made software was used for the FNS computations. The "stepwiseness factor" $\sigma$ and the "spikiness factor" $S\left(L_{0}^{-1}\right)$ were used as the surface parameters for the comparison of the surface morphology at different atherosclerosis stages. It should be noted that, while FNS power spectra do demonstrate the intrinsic periodicity of collagenous structures (D-period), in general, FNS parameters have no direct reference to the measurable characteristics of extracellular matrix, such as the collagen content or fibrils' dimensions. However, correlations in the chaotic surface profiles (revealed by the FNS analysis) appear rather sensitive to the structure ordering/disordering due to various processes, ${ }^{15,16}$ and thus, the FNS parameters derived from such correlations may be used as markers for a comparative analysis of the extracellular matrix structure.

\subsection{Region of Interest Selection}

The ROIs for the quantitative evaluation of CP OCT, SHG, and AFM images were selected manually. These ROIs had a specific size for each imaging modality: $2 \times 0.3 \mathrm{~mm}$ for CP OCT, $0.2 \times$ $0.1 \mathrm{~mm}$ for SHG, and $6 \times 6 \mu \mathrm{m}$ for AFM. The ROIs of CP OCT, SHG, and AFM were specifically chosen within the upper part of an atherosclerotic plaque, namely, in the fibrous cap or intima of different stages of atherosclerosis development: thickened intima (stage I), thickened intima with the deposition of lipids (stages II/III), thickened fibrous cap (stage IV), a thin fibrous cap (stage Va), a fibro-calcified cap (stage $\mathrm{Vb}$ ), and a very thick fibrous cap (stage Vc).

The manual ROI selection was used with the understanding that if a significant difference between these areas was observed, the next research stage would be moving toward more automated processing to develop an automatic procedure of plaque differentiation in the future.

\subsection{Statistical Processing}

The data distribution normality was verified using the Kolmogorov-Smirnov criterion. The Kolmogorov-Smirnov test quantifies the distance between the empirical cumulative distribution function of the sample and the cumulative distribution function of the reference distribution; in this case, the reference is the Gaussian normal distribution. The null hypothesis for the Kolmogorov-Smirnov test is that the distribution is different from normal, and it is rejected if the $p$ value (probability that the difference is just a random chance) is higher than 0.05 [in our case, it is found to be 0.2 for the IDF and average $\Delta n$ values for two plaque stages (IV and Va)]. Therefore, parametric statistical tests were used. All the results were expressed as mean $\pm \mathrm{SD}$. 
The statistical significance of differences in effective $\Delta n$, IDF, and isotropy indices between the stages IV and Va was calculated by the student's $t$-test. The multiple comparisons with the Bonferroni correction were used, in particular to compare stage I with all the other stages. In all cases, the differences were considered statistically significant when $p<0.05$. The Spearman's correlation coefficient $(r)$ was calculated to determine the correlation among the isotropy index, effective birefringence, and DF.

The statistical data processing was done in MS Excel 2010 with a public domain software plugin for statistical analysis STATISTICA 10 (StatSoft, Inc., Tulsa, Oklahoma) and the Prism v6 software (GraphPad Software, La Jolla, California).

\section{Results}

\subsection{Visual Assessment of Cross-Polarization Optical Coherence Tomography, Histology, Second- Harmonic Generation, and Atomic Force Microscopy Images}

CP OCT, histology, SHG, and AFM were used previously to separately assess the content, spatial, and structural organization of collagen fibers in atherosclerotic plaques. ${ }^{11,15}$ In this study, these methods are combined for a parallel comparative evaluation of the different stages of plaque development.

The comparison of CP OCT images of atherosclerotic plaques at different stages of atherosclerosis (I, II/III, IV, Va, $\mathrm{Vb}$, and $\mathrm{Vc}$ stages) reveals a tendency for violation of the layered structure in the vessel wall [Figs. 2(a)-2(f)]. Second, the changes in anisotropic structures, such as collagen fibers, influence the CP channel brightness [the lower part of the image in Figs. 2(a)-2(f)]. It should be noted that the changes in packaging density, microstructural organization, and the geometric orientation of collagen fibers in the intima/fibrous cap are sensitive markers for the progression of atherosclerosis [Figs. 2(g)-2(l), yellow rectangle] and identification of vulnerable (prone to rupture) plaques [Fig. 2(j)]. For a detailed analysis of the fibrous patterns of the intima or fibrous caps, SHG and AFM images were obtained to compare them with the corresponding $\mathrm{CP}$ OCT data.

CP OCT image in the $\mathrm{CP}$ channel at the stage I of a plaque demonstrated conservation of the vessel wall layered structure visible as thin horizontal layers with a higher signal level in the

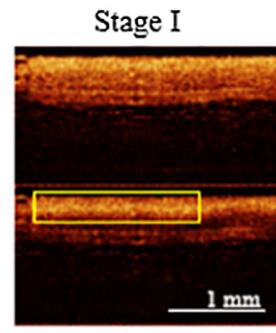

(a)

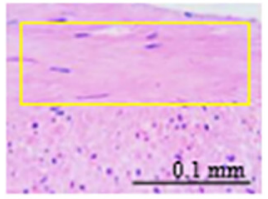

(g)

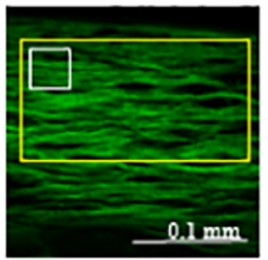

(m)

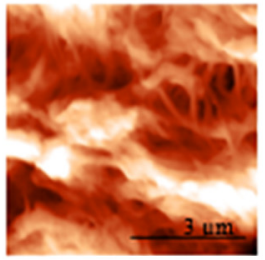

(s)

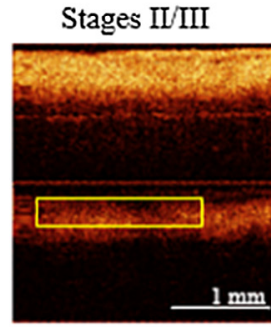

(b)

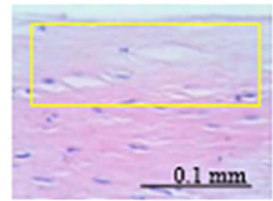

(h)

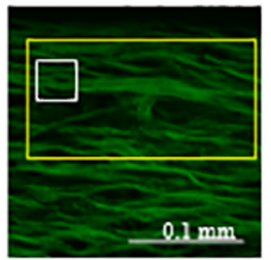

(n)

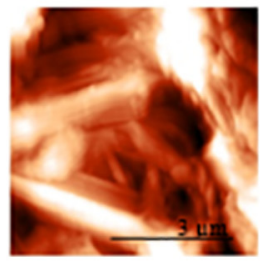

$(\mathrm{t})$

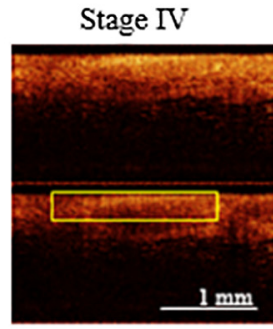

(c)

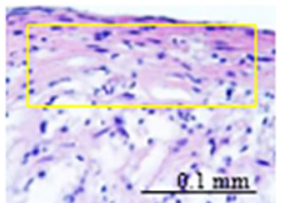

(i)

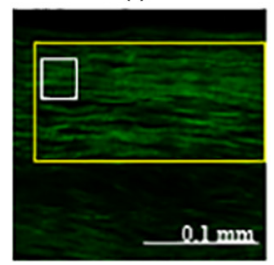

(o)

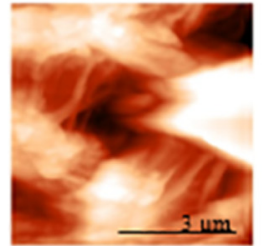

(u)

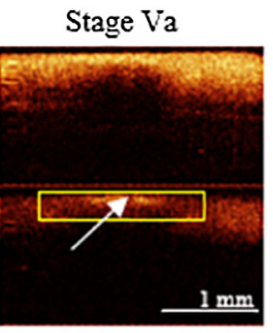

(d)

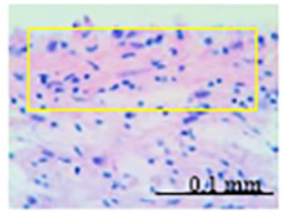

(j)

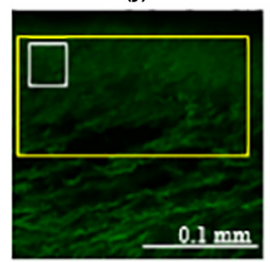

(p)

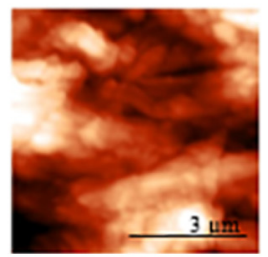

(v)

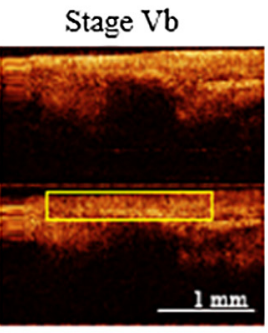

(e)

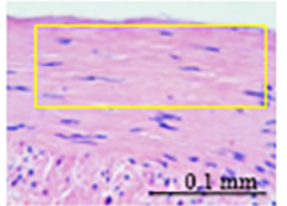

(k)

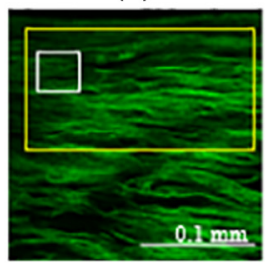

(q)

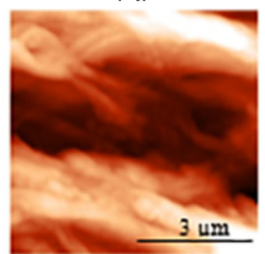

(w)

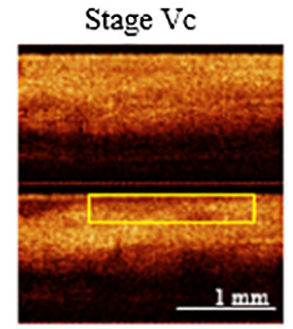

(f)

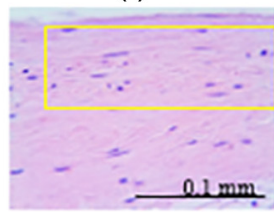

(I)

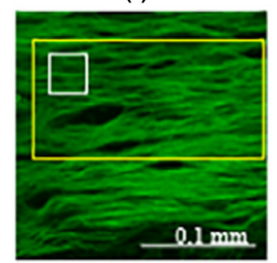

(r)

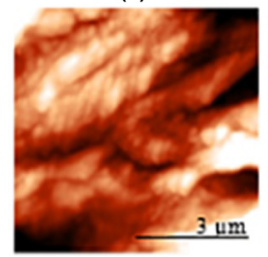

(x)

Fig. 2 Visualisation of different stages of a plaque development by CP OCT, histology, SHG, and AFM. (a-f) CP OCT images (upper panel = copolarization channel, lower panel = CP channel), (g-l) histological slides stained with H\&E, (m-r) SHG images, and (s-x) AFM images. The rectangles in the CP OCT images indicate the area covered by histological slides and SHG images, respectively. The arrow points to accumulations of foam and inflammatory cells at the border of the fibrous cap and the lipid core. The square in the SHG images indicates the area studied by AFM. 
thickened intima [Fig. 2(a), rectangle]. The intima is represented predominantly by collagen fibers [Fig. 2(g)], which are well oriented along the vessel wall, demonstrate an intensive SHG signal [Fig. 2(m)], and have a typical banded structure of collagen fibrils, forming a basket-weave pattern at the AFM image [Fig. 2(s)].

With the development of atherosclerosis, the collagen fibrils' packing is changed and they are covered with an amorphous material (protein-proteoglycan complexes) as can be seen at the AFM images [Figs. 2(t)-2(x)]. The corresponding SHG images demonstrate a gradual change of the packing density and organization of collagen fibers [Figs. 2(n)-2(r)]. It is especially important to note the strong disorganization of collagen fibers in the fibrous caps of vulnerable plaques (stage Va) as seen from histology [Fig. 2(j)], SHG [Fig. 2(p)], and AFM [Fig. 2(v)] images.

The corresponding CP OCT images can be analyzed for the detected changes in organization/disorganization of collagen fibers by the OCT signal level in the CP channel [Figs. 2(a)-2(f)]. The deposition of lipids on the surface or inside the bulk of the intima as the lipid strips (stages II/III) produces almost no CP signal at the top of the image, but then the CP brightness increases, possibly because of the fiber birefringence coexisting with the lipid [Fig. 2(b), rectangle]. In the case of stable plaques (stages IV, Vb, and Vc), a high CP brightness is observed near the surface from a fibrous cap over the lipid core [Figs. 2(c), 2(e), 2(f), yellow rectangle, respectively]. Specific for a vulnerable plaque (stage $\mathrm{Va}$ ) is a highly heterogeneous OCT signal, quickly decreasing with the depth in the copolarization channel [the upper part of the image in Fig. 2(d)]. In the CP channel, this area shows a weak heterogeneous OCT signal. This finding indicates the prevalence of disrupted fibers in the fibrous cap and can serve as an indicator of plaque instability [Fig. 2(d), rectangle]. The cell clusters located between the lower part of the fibrous cap and the surface of the lipid core are visible in the CP channel as "bright spots" [arrow, lower panel, Fig. 2(d)].

Thus, one may note that the CP channel in CP OCT plaque images provides an additional contrast for the assessment of the collagen fibers' condition.

To provide a more objective assessment of the main structural components of plaques at different development stages, a quantitative analysis of the CP OCT, SHG, and AFM images was proposed (Sec. 3.2).

\subsection{Quantitative Assessment of the Cross- Polarization Optical Coherence Tomography, Second-Harmonic Generation, and Atomic Force Microscopy Images}

Quantitative characteristics derived from the different imaging modalities data for different plaque development stages are shown in Fig. 3. The IDF calculation [Fig. 3(a)] revealed a statistically significant difference between a plaque at the initial stage of development (stage I), a vulnerable plaque (stage Va), and a fibroatheroma with severe fibrosis (stage Vc). The IDF allows reliable differentiation between stable (stage IV) and vulnerable (stage Va) plaques $(0.46 \pm 0.21$ against $0.09 \pm 0.04 ; p<0.001)$ origination from significant difference in spatial organization collagen, although the structure manifested by thin fibrous cap and a lipid core is similar. Both types of plaques have thin fibrous caps and a lipid core, but a different spatial organization of collagen in the fibrous cap above the lipid core, thus, they are hard to be distinguished by traditional diagnostic techniques. The IDF decrease at Va stage is consistent with the histologically known significant disorganization of the collagen fibers' orientation.

The results of the average effective $\Delta n$ calculation from CP OCT images of plaques at different stages are shown in Fig. 3(b). The average $\Delta n$ value is significantly higher in the slightly thickened intima at the initial stage of a plaque (stage I) as compared to the following stages of plaque development $(p<0.05)$. At the same time, the high values of the average $\Delta n$ for stages $\mathrm{I}, \mathrm{IV}, \mathrm{Vb}$, and $\mathrm{Vc}$ may indicate the presence of a large number of ordered anisotropic structures (collagen fibers) distributed homogeneously in the fibrous cap of a stable plaque. It was found that the average $\Delta n$ of the fibrous cap of a stable plaque (stage IV) amounting $(0.47 \pm 0.10) \cdot 10^{-3}$ is higher than that for the disorganized fibers of a vulnerable plaque (stage Va) reaching $\left[(0.25 \pm 0.07) \cdot 10^{-3} ; p<0.001\right]$. However, the Va plaque cap is too thin to have a measurable (by CP OCT) birefringence, so this is considered as an evidence-based observation rather than a statement about the CP OCT measurement of the real $\Delta n$.

The low values of the IDF and average $\Delta n$ at stage Va may indicate the predominance of disorganized collagen fibers in the fibrous cap. Another explanation for such a decrease of the CP channel brightness is that ROI included a lipid strip on the surface of the intima, and lipid (for example at the stages II/III) is not capable of providing $\mathrm{CP}$ backscattering.
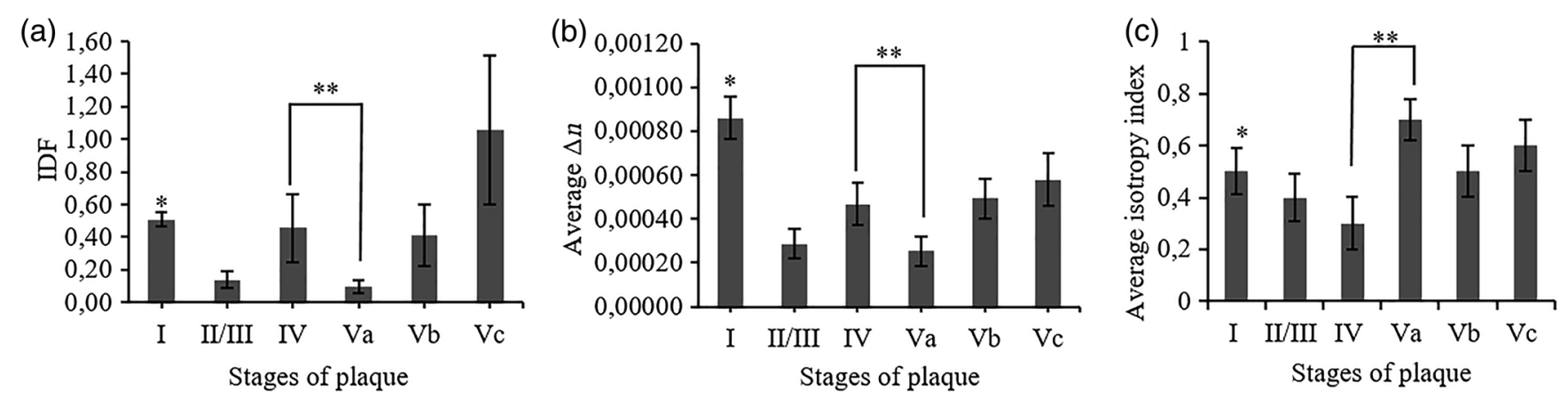

Fig. 3 (a) IDF, (b) average $\Delta n$, and (c) average isotropy index for different stages of plaque development. Asterisk $\left(^{*}\right)$ indicates a statistically significant difference between stages I and all the subsequent plaque development stages, $\mathrm{p}<0.05$ (Bonferroni posthoc test), double asterisk $\left(^{\star *}\right)$ indicates a statistically significant differences between stable (IV) and vulnerable (Va) plaque stages, $p<0.001$ (student's $t$-test). 
The calculation of the average isotropy index from SHG images was used as an alternative method to analyze the collagen fibers order in the intima/fibrous cap. The calculated values of the average isotropy index are shown at the histogram in Fig. 3(c). As expected, the average isotropy index has lower values for stable (stages I, II/III, IV, and Vb) plaques than that for vulnerable (stage Va) plaques, demonstrating a higher alignment of collagen fibers in a stable plaque in contrast to a more isotropic distribution within a vulnerable plaque [Fig. 3(c)]. The low values of the isotropy index for stages II/III indicate a presence of organized collagen fibers and it appears difficult to determine a correlation with CP OCT showing the low value of the average $\Delta n$ and IDF. This occurs due to the fact that the SHG signal in tissue is observed exclusively in densely packed, organized structures, such as collagen, which still retains organization in between clusters of lipids at this stage of plaque development.

A statistically significant difference $(p<0.05)$ between the average isotropic indices of disorganized fibers of fibrous caps in a vulnerable plaque (stage $\mathrm{Va}$ ) and of all the other stages of a plaque (stages I, II/III, IV, Vb, and Vc) was found. In addition, a statistically significant difference exists between the average value of isotropy index of stable (IV) and vulnerable (Va) plaque stages $(0.30 \pm 0.10$ against $0.70 \pm 0.08 ; p<0.001)$, which supports the interpretation of the OCT signal decrease in CP OCT images of a vulnerable atherosclerotic plaque [Fig. 3(a), 3(b)].

The Spearman's correlation coefficient shows a high negative correlation between the average isotropy index derived from SHG images and the average $\Delta n$, calculated from CP OCT images: for a stable (stage IV) plaque $r=-0.81$ and for a vulnerable (stage Va) plaque $r=-0.94(p<0.001)$. The correlation coefficients between the average isotropy index and IDF are as follows: for a stable plaque $r=-0.83$ and for a vulnerable plaque $r=-0.89(p<0.001)$. This correlation is shown for the first time and demonstrates the high degree of concordance between the tissue's ability to exhibit birefringence/CP backscattering. Consequently, basing on the CP OCT images, a structural organization of collagen fibers in tissues can be assessed. Moreover, by quantitative characterization of organization of collagen fibers through the IDF and average $\Delta n$ analysis, higher values were revealed for stable plaques as compared to vulnerable plaques.

The total averaged values of the peak-to-valley roughness and FNS parameters in the AFM images are represented in Fig. 4. The gradual reduction of the peak-to-valley roughness $h_{m}$ with the severity of atherosclerosis stages indicates that the surface becomes smoother [Fig. 4(a)]. The high values of FNS $\left[\sigma\right.$ and $\left.S\left(L_{0}^{-1}\right)\right]$ parameters in the area of the norm (stage I) are likely associated with a wavy structure of collagen fibers in the intima, which is manifested as "holes" at the cross sections of a sample, making the tissue surface looser. For all the following pathological stages of plaque development (stages II/ III, IV, Va, Vb, and Vc), both FNS parameters have lower values, but at the later plaque stages, the parameters' values increase, which is associated with disordering of collagen fibrils [Figs. 4(b) and 4(c)]. The growth of the $S\left(L_{0}^{-1}\right)$ peaks with the transition from the early stage to the later stages of atherosclerosis may be caused by the increased proportion of uncoated collagen fibrils and by gaps in the collagen network, resulting in more explicit differences in the heights of profiles [Fig. 4(c)]. At the stage $\mathrm{Vc}$ of atherosclerosis development, the FNS parameters again decrease, and this can be explained by the formation of a smoother surface due to the thick coverage of collagen fibrils and fibers with unstructured proteins. At the same time, the characteristic frequencies in the power spectra of the last stages are either weakened (stage Vc) or almost disappear (stage Va). ${ }^{15}$ It should be noted that there is a rather noticeable error in the measured values of the $S\left(L_{0}^{-1}\right)$ parameter due to the high degree of surface heterogeneity of the studied samples.

Thus, the FNS analysis of the surface topography, which indicates the disordering of collagen fibers with the development of atherosclerosis, also supports the reduction in the polarization properties at CP OCT images obtained for the fibrous cap of a vulnerable atherosclerotic plaque.

\section{Discussion}

This study shows the potential of CP OCT in the assessment of collagen fibers condition in an atherosclerotic plaque at different stages of atherosclerosis. Currently, the majority of OCT criteria for differential diagnosis of atherosclerotic plaques are based on qualitative visual interpretation, including the signal level and heterogeneity, shape and size of different microstructures in a plaque, the level of birefringence indicative of the orientation, and the level of structural collagen organization in the PS OCT images. ${ }^{7,25,26}$ The quantitative analysis of CP OCT images has a potential to improve the efficacy of vulnerable plaque detection.

The current study presents a more comprehensive evaluation of all stages of atherosclerotic plaque development using $\mathrm{CP}$ OCT, histology, nonlinear microscopy, and AFM to analyze the specific features of the collagen structure at multiple spatial levels (Figs. 2, 3, and 4).
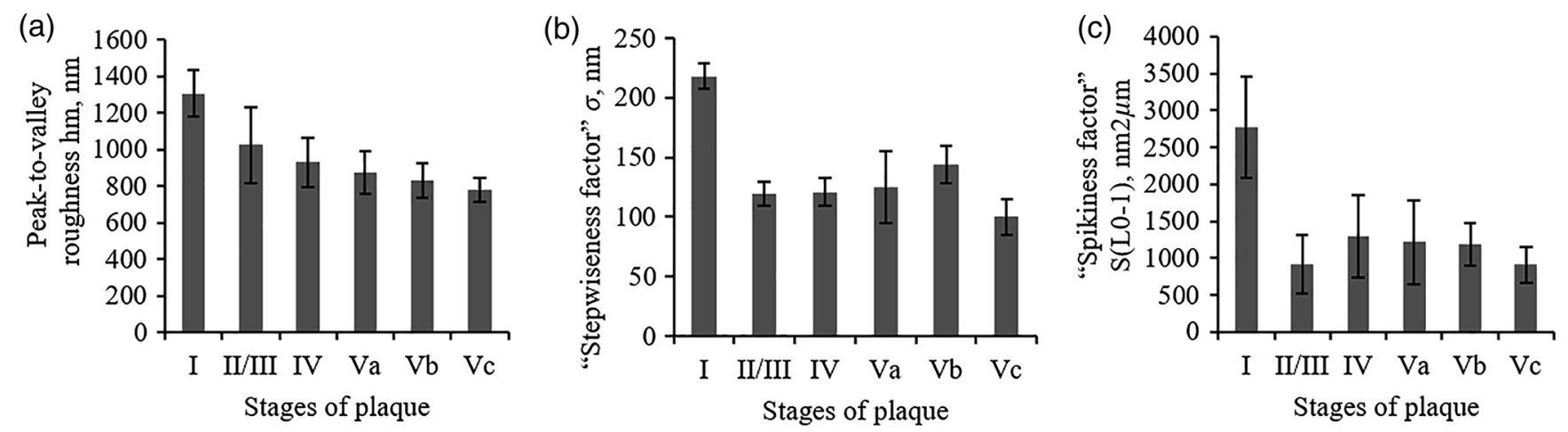

Fig. 4 (a) Histograms of peak-to-valley roughness $h_{m}$ and FNS parameters of AFM images of different stages of plaque development, (b)"stepwiseness factor" $\sigma$, and (c) "spikiness factor" $S\left(L_{0}^{-1}\right)$. 
The FFT analysis of the SHG signal in this study allowed us to differentiate not only between the normal arterial wall and plaque deposition, but also between all the successive stages of plaque development [Fig. 3(c)] that was not shown previously. AFM visualization of fibrils inside collagen fibers at a nano/micrometer spatial scale is an informative method for assessing disordering of the collagen structures in fibrous caps during the progression of atherosclerosis plaques and can help to better understand the starting mechanisms of capsule rupture (Fig. 4). AFM modalities, including imaging and morphology quantification using FNS, demonstrate the generalized character of changes taking place in the upper fibrous layer with atherosclerosis progression. The involvement of the entire hierarchical network of collagen into the process invariably has an impact on the mechanical properties of the fibrous cap and the corresponding risks.

Unlike OCT, endoscopic and intravascular applications of SHG and AFM microscopy still remain unavailable for translation into the clinical practice, and in this paper, they are considered as tools to enhance and validate our understanding of the plaque microstructure and the origin of the CP OCT observed features.

The proposed quantitative approach to characterization of collagen fibers in the blood vessels' walls by CP OCT consists in calculation if IDF and average effective $\Delta n$. The approach may become a basis for differentiation of stages of plaques development in order to identify their stability. This study revealed that increased $\mathrm{CP}$ backscattering correlated with abundant thick collagen fibers of the intima/fibrous cap, suggesting that the detection of a higher level of signal in the CP channel of CP OCT images implied the increased stability of a plaque.

The quantitative assessment of CP OCT images could become a potentially powerful minimally invasive method for vulnerable plaques detection. Calculation of the IDF and the average effective $\Delta n$ provides important information about stable and vulnerable plaques' fibrous cap, which is in agreement with the results of SHG and AFM. In this study, a statistically significant correlation was observed between the coronary artery collagen changes assessed by CP OCT and SHG. Atherosclerosis involves inflammation in which the fibers' structure in the intima/fibrous cap is less organized. It is reasonable to expect that the IDF reflects these changes, and the differences between stable (stage IV) and vulnerable (stage Va) plaques are found to be significant [Fig. 3(a)]. A simultaneous calculation of the effective birefringence $(\Delta n)$ in the ROI provides an additional information about the structural organization changes in the intima/fibrous cap [Fig. 3(b)]. So, as stable plaques with a thickened fibrous cap tend to increase the concentration and density of their fibers, their order, and level of organization, they exhibit a higher level of signal in the CP channel. By contrast, a vulnerable plaque should have a reduced $\mathrm{CP}$ backscattering, which was confirmed through the quantitative assessment of CP OCT images. The effective birefringence $(\Delta n)$ calculated from the CP OCT images for the main stages of atherosclerosis is consistent with the data in Ref. 6. Furthermore, the level of CP backscattering shows a statistically significant difference between the stable (stage IV) and vulnerable (stage Va) plaques. These stages IV and Va can be similar in size and do not lead to visible narrowing in the vessel lumen. They are therefore difficult to be differentiated by means of common intravascular imaging techniques. ${ }^{27}$ However, the internal structure of both the fibrous cap and the lipid core for these stages is completely different morphologically.
When calculating the IDF and the average $\Delta n$, we used an approach involving a manual, subjective selection of the ROI in the CP OCT images. Thus, the results of the CP OCT images processing might be considered as a measure of polarization properties of stable and vulnerable atherosclerotic plaques, rather than the criteria for independent plaque diagnostics by CP OCT technique. Nevertheless, the developed approach to a quantitative assessment of polarization properties of atherosclerotic plaques can provide a basis for a future development of automated evaluation of plaques by CP OCT, including detection of vulnerable plaques in vivo.

Thus, we believe that the presented results may stimulate a future development of the CP OCT technique in the clinical practice to expand the diagnostic capabilities of OCT, particularly, in the clinical in vivo vulnerable plaques detection.

\section{Conclusions}

The visual and quantitative analysis of the CP OCT images confirmed the presence of intima/fibrous cap $\mathrm{CP}$ backscatter properties in the human coronary artery, influenced by a well-defined spatial and structural organization of the collagen matrix. Collagen fibers undergo significant changes as a result of pathological processes, which affect their polarization properties as detected and quantified by CP OCT. The ability of SHG and AFM microscopy to selectively visualize collagen fibers and fibrils and quantitatively assess their physical properties allowed tracking the extent of the collagen disruption at all levels of its organization and provided a better understanding of the connection between the collagen matrix state and the CP backscatter in the CP channel of CP OCT. The IDF and average effective $\Delta n$ calculated from CP OCT images can be applied to detect vulnerable plaques. CP OCT has a potential to provide an objective, minimally invasive characterization of the collagen matrix and, thus, track the atherosclerotic plaques progression.

\section{Disclosures}

The authors have no relevant financial and competing interests in the manuscript and no other potential conflicts of interest.

\section{Acknowledgments}

The medical and biological part of this work, as well as the analysis of the efficiency of algorithms for image processing and selection of optimal quantitative characteristics, were supported by the Russian Science Foundation, Grant No. 14-1500538 (EVG, MYuK, VVD, EBK, LBT, SLK, GVB, and NDG). The numerical image processing was supported by the grant of the Russian Foundation for Basic Research No. 15-32-20250. The development of the CP OCT equipment was provided by the Ministry of Education and Science of the Russian Federation, Grant No. 14.B25.31.0015.

\section{References}

1. R. Virmani et al., "Pathology of the vulnerable plaque," J. Am. Coll. Cardiol. 47, C13-C18 (2006).

2. P. Libby, P. M. Ridker, and G. K. Hansson, "Progress and challenges in translating the biology of atherosclerosis," Nature 473, 317-325 (2011).

3. Y. Fukumoto et al., "Statins alter smooth muscle cell accumulation and collagen content in established atheroma of watanabe heritable hyperlipidemic rabbits," Circulation 103, 993-999 (2001).

4. P. K. Shah et al., "Human monocyte-derived macrophages induce collagen breakdown in fibrous caps of atherosclerotic plaques. Potential 
role of matrix-degrading metalloproteinases and implications for plaque rupture," Circulation 92, 1565-1569 (1995).

5. E. Falk, P. K. Shah, and V. Fuster, "Coronary plaque disruption," Circulation 92, 657-671 (1995).

6. W. C. Kuo et al., "Assessment of arterial characteristics in human atherosclerosis by extracting optical properties from polarization-sensitive optical coherence tomography," Opt. Express 16, 8117-8125 (2008).

7. S. Nadkami et al., "Polarization-sensitive optical coherence tomography for the analysis of collagen content in atherosclerotic plaques," Circulation 112, 679 (2005).

8. S. D. Giattina et al., "Assessment of coronary plaque collagen with polarization sensitive optical coherence tomography (PS-OCT)," Int. J. Cardiol. 107, 400-409 (2006).

9. V. M. Gelikonov and G. V. Gelikonov, "New approach to cross-polarized optical coherence tomography based on orthogonal arbitrarily polarized modes," Laser Phys. Lett. 3, 445-451 (2006).

10. J. M. Schmitt and S. H. Xiang, "Cross-polarized backscatter in optical coherence tomography of biological tissue," Opt. Lett. 23, 1060-1062 (1998).

11. E. V. Gubarkova et al., "Multi-modal optical imaging characterization of atherosclerotic plaques," J. Biophotonics 9, 1009-1020 (2016).

12. A. Zoumi et al., "Imaging coronary artery microstructure using secondharmonic and two-photon fluorescence microscopy," Biophys. J. 87, 2778-2786 (2004).

13. R. Cicchi et al., "Characterization of collagen and cholesterol deposition in atherosclerotic arterial tissue using non-linear microscopy," J. Biophotonics 7, 135-143 (2014).

14. S. Seidenari et al., "Multiphoton laser tomography and fluorescence lifetime imaging of basal cell carcinoma: morphologic features for non-invasive diagnostics," Exp. Dermatol. 21, 831-836 (2012).

15. P. S. Timashev et al., "Atomic force microscopy study of atherosclerosis progression in arterial walls," Microscopy and Microanalysis 22, 311325 (2016)

16. U. Mirsaidov et al., "Analytical method for parameterizing the random profile components of nanosurfaces imaged by atomic force microscopy," Analyst 136, 570-576 (2011).

17. H. C. Stary et al., "A definition of advanced types of atherosclerotic lesions and a histological classification of atherosclerosis: a report from the Committee on Vascular Lesions of the Council on Arteriosclerosis, American Heart Association" Arterioscler. Thromb. Vasc. Biol. 15, 1512-1531 (1995).

18. V. Gelikonov, G. Gelikonov, and P. Shilyagin, "Linear-wavenumber spectrometer for high-speed spectral-domain optical coherence tomography," Opt. Spectrosc. 106, 459-465 (2009).

19. A. A. Moiseev et al., "Noniterative method of reconstruction optical coherence tomography images with improved lateral resolution in semitransparent media," Laser Phys. Lett. 10, 125601 (2013).

20. Z. Hu and A. M. Rollins, "Fourier domain optical coherence tomography with a linear-in-wavenumber spectrometer," Opt. Lett. 32, 35253527 (2007).

21. E. Kiseleva et al., "Differential diagnosis of human bladder mucosa pathologies in vivo with cross-polarization optical coherence tomography," Biomed. Opt. Express 6, 1464 (2015).

22. E. V. Gubarkova et al., "Cross-polarization optical coherence tomography in evaluation of atherosclerotic plaque structure," Modern Technol. Med. 5, 45-55 (2013).

23. R. D. Andrews and J. F. Rudd, "Birefringence changes during retraction of oriented polystyrene monofilaments. I. Changes of average birefringence," J. Appl. Phys. 27, 990-995 (1956).

24. R. Cicchi et al., "Scoring of collagen organization in healthy and diseased human dermis by multiphoton microscopy," J. Biophotonics $\mathbf{3}$, 34-43 (2009).

25. W. C. Kuo et al., "Polarization-sensitive optical coherence tomography for imaging human atherosclerosis," Appl. Opt. 46, 2520-2527 (2007).

26. S. K. Nadkami et al., "Evaluation of collagen in atherosclerotic plaques: the use of two coherent laser-based imaging methods," Laser Med. Sci. 24, 439-445 (2009).

27. G. J. Tearney et al., "Consensus standards for acquisition, measurement, and reporting of intravascular optical coherence tomography studies: a report from the International Working Group for Intravascular Optical Coherence Tomography Standardization and Validation," J. Am. Coll. Cardiol. 59, 1058-1072 (2012).
Ekaterina V. Gubarkova received her $\mathrm{PhD}$ in biology and is a junior researcher at the Laboratory of Studying Optical Structure of Biotissue, the Scientific Research Institute of Biomedical Technologies (Nizhny Novgorod State Medical Academy). She graduated from the N.I. Lobachevsky State University of Nizhny Novgorod as a specialist in biophysics. Her research interests include optical imaging of biotissues, cross-polarization optical coherence tomography, polarization, and confocal laser-scanning microscopy. She is the author of 10 research publications.

Mikhail Yu. Kirillin received his DrSc in technology and $\mathrm{PhD}$ in physics and mathematics, and is a senior research fellow at the Institute of Applied Physics RAS. He graduated with honors from M.V. Lomonosov Moscow State University. His scientific interests include optics of biotissues and other scattering media, optical tomographic systems including optical coherence and diffusive modalities, optical clearing and contrast enhancement techniques, and Monte Carlo simulations of light transport in scattering media. $\mathrm{He}$ is the author of more than 100 research publications.

Varvara V. Dudenkova is a graduate student of the N. I. Lobachevsky State University of Nizhny Novgorod and a junior researcher of the Laboratory of Studying Optical Structure of Biotissue, the Scientific Research Institute of Biomedical Technologies (Nizhny Novgorod State Medical Academy). She graduated from the N.I. Lobachevsky State University of Nizhny Novgorod as a specialist in radiophysics. Her research interests include fluorescent microscopy and holography for biomedicine. She is the author of 10 research publications.

Peter S. Timashev received his $\mathrm{DrSc}$ and his $\mathrm{PhD}$ in chemistry and is a senior research fellow at Institute of Photonic Technologies, Research Center "Crystallography and Photonics" RAS and Director of Biomaterials Department in Institute for Regenerative Medicine, I. M. Sechenov First Moscow State Medical University. He graduated from Moscow Institute of Fine Chemical Technology as a specialist in chemistry. His fields of scientific interests include supercritical fluids, atomic force microscopy (AFM), and regenerative medicine. He is the author of 70 research publications.

Svetlana L. Kotova received her PhD in chemistry and is a senior research scientist at the Laboratory of Modified Polymers, Department of Polymers, N.N. Semenov Institute of Chemical Physics. She graduated from the M.V. Lomonosov Moscow State University as a specialist in chemistry. Her research interests include AFM of biological and polymer materials. She is the author of 36 research publications.

Elena B. Kiseleva received her PhD in biology and is a research fellow at the Laboratory of Studying Optical Structure of Biotissues of the Scientific Research Institute of Biomedical Technologies (Nizhny Novgorod State Medical Academy). She graduated from the N.I. Lobachevsky State University of Nizhny Novgorod as a specialist in biophysics. Her fields of scientific interests include optical imaging of biotissues and collagen state change in pathologic tissues and in course of radiation therapy. She is the author of 43 research publications.

Lidia B. Timofeeva received her $\mathrm{PhD}$ in biology and is an assistant at the Department of Histology, Nizhny Novgorod State Medical Academy. She graduated from the N.I. Lobachevsky State University of Nizhny Novgorod as a biologist. Her scientific interests include investigation biological tissues morphology by light microscopy. She is the author of 12 research publications.

Galina V. Belkova received her $\mathrm{PhD}$ in chemistry and is a senior research scientist at the Laboratory of Modified Polymers, Department of Polymers, N.N. Semenov Institute of Chemical Physics. She graduated from Moscow State University of Fine Chemical Technologies as a specialist in chemical engineering. Her research interests include AFM of biological and polymer materials. She is the author of 22 research publications.

Anna B. Solovieva received her DrSc in chemistry and is the head of the Laboratory of Modified Polymers, the Department of Polymers, N.N. Semenov Institute of Chemical Physics. She graduated from the M.V. Lomonosov Moscow State University as a 
specialist in chemistry. Her research interests include polymer chemistry and physics, structure of polymers and multicomponent polymer systems, photochemistry of porphyrins, and other biologically active substances. She is the author of more than 250 research publications.

Alexander A. Moiseev received his $\mathrm{PhD}$ in physics and mathematics and is a research scientist at the Laboratory of Highly Sensitive Optical Measurements, the Institute of Applied Physics of the Russian Academy of Sciences. He received his master's degree in physics from the N.I. Lobachevsky State University of Nizhny Novgorod and his PhD in radiophysics from the Institute of Applied Physics of the RAS. His fields of scientific interests include optical coherence tomography and subdiffraction fluorescence microscopy. $\mathrm{He}$ is the author of 16 research publications.

Gregory V. Gelikonov received his $\mathrm{PhD}$ in physics and mathematics and is the head of the Laboratory of Highly Sensitive Optical Measurements, the Institute of Applied Physics, the Russian Academy of Sciences. He graduated from the N.I. Lobachevsky State University of Nizhny Novgorod as a specialist in radiophysics and electronics. His scientific interests include optical coherence tomography, fiber-optical interferometry, highly sensitive optical measurements, bioimaging, fiber optics, and low-coherence interferometry. $\mathrm{He}$ is the author of 73 research publications.

llya I. Fiks received his $\mathrm{PhD}$ in physics and mathematics and is a Research fellow at the Institute of Applied Physics RAS. He graduated from the Computational Mathematics and Cybernetics Department of N.I. Lobachevsky State University of Nizhny Novgorod. His scientific interests include numerical methods, inverse problems, light propagation in heterogeneous media, image processing and classification, and reconstruction techniques. $\mathrm{He}$ is the author of more than 30 research publications.

Felix I. Feldchtein received his $\mathrm{PhD}$ in quantum electronics and is a scientist at the Laboratory "Optical Coherent Tomography" of the Scientific Research Institute of Biomedical Technologies (Nizhny Novgorod State Medical Academy). He graduated from the Radiophysical Department of N.I. Lobachevsky State University of Nizhny Novgorod. His research interests include nonlinear optics, laser-matter interaction, optical coherence tomography, laser surgery, and dentistry. $\mathrm{He}$ is the author of more than 170 research publications and more than 30 international issued and pending patents.

Natalia D. Gladkova received her DSc in medicine, professor, Laureate of the State Prize of the Russian Federation, and deputy head of the director for Research Work of the Scientific Research Institute of Biomedical Technologies (Nizhny Novgorod State Medical Academy). She is the author of more than 200 research publications on optical coherence tomography in endoscopy, oncology, stomatology, and dermatology. She is the author of the monograph "Optical coherence tomography among medical imaging modalities. A course of lectures" (in Russian, 2005) and editor-in-chief of Handbook of Optical Coherence Tomography (in Russian, 2007). 